\title{
Public health policy pillars for the sustainable elimination of zoonotic schistosomiasis
}

\author{
Eva Janoušková ${ }^{1, *}$, Jessica Clark ${ }^{2,3}$, Olumayowa Kajero ${ }^{1}$, Sergi Alonso ${ }^{2,3}$, \\ Poppy H. L. Lamberton ${ }^{2,3}$, Martha Betson ${ }^{1}$ and Joaquín M. Prada ${ }^{1}$ \\ ${ }^{1}$ School of Veterinary Medicine, University of Surrey, Guildford, United Kingdom \\ ${ }^{2}$ Institute of Biodiversity, Animal Health and Comparative Medicine, University of \\ Glasgow, Glasgow, United Kingdom \\ ${ }^{3}$ Wellcome Centre for Integrative Parasitology, University of Glasgow, Glasgow, \\ United Kingdom
}

Correspondence*:

Eva Janoušková

e.janouskova@surrey.ac.uk

2 Article type: Perspective

3 Number of words in the abstract: 204

4 Number of words in the main text: 2, 458

5 Number of figures: 1

6 Number of tables: 0

7 Language: British English

\section{ABSTRACT}

Schistosomiasis is a parasitic disease acquired through contact with contaminated freshwater. The definitive hosts are terrestrial mammals, including humans, with some Schistosoma species crossing the animal-human boundary through zoonotic transmission. An estimated 12 million people live at risk of zoonotic schistosomiasis caused by Schistosoma japonicum and Schistosoma mekongi, largely in the World Health Organization's Western Pacific Region and in Indonesia. Mathematical models have played a vital role in our understanding of the biology, transmission, and impact of intervention strategies, however, these have mostly focused on non-zoonotic Schistosoma species. Whilst these non-zoonotic-based models capture some aspects of zoonotic schistosomiasis transmission dynamics, the commonly-used frameworks are yet to adequately capture the complex epi-ecology of multi-host zoonotic transmission. However, overcoming these knowledge gaps goes beyond transmission dynamics modelling. To improve model utility and enhance zoonotic schistosomiasis control programmes, we highlight three pillars that we believe are vital to sustainable interventions at the implementation (community) and policy-level, and discuss the pillars in the context of a One-Health approach, recognising the interconnection between humans, animals and their shared environment. These pillars are: (1) human and animal epi-ecological understanding; (2) economic considerations (such as treatment costs and animal losses); and (3) sociological understanding, including inter- and intra-human and animal interactions. 


\section{INTRODUCTION}

Neglected Tropical Diseases (NTDs) predominantly affect communities in low- and middle-income countries and impose a significant human, economic and social burden, thus perpetuating a cycle of poverty. Schistosomiasis is caused by infection with parasitic worms of the genus Schistosoma. An estimated 240 million people are infected 1 and the disease is classified by the World Health Organization (WHO) as an NTD. The main types of schistosomes responsible for human disease are Schistosoma haematobium, causing urogenital disease, and S. mansoni, S. japonicum, and S. mekongi causing intestinal disease. They differ in geographical distribution and the host species they infect. Schistosoma haematobium and $S$. mansoni mainly infect humans as the definitive host. Schistosoma japonicum (found in the Philippines, China, Indonesia), and S. mekongi (Cambodia and Laos) on-the-other-hand use both human and non-human mammals as definitive hosts, driving zoonotic transmission across the human-animal boundary. Over 12 million people are estimated at risk of zoonotic infection in Asia with three million requiring treatment. Though vital to transmission, the number of animals at risk is generally not reported ${ }^{2}$ (1). The life-cycle of zoonotic schistosomes is maintained by human and animal contact with contaminated freshwater sources, where the intermediate hosts (species-specific freshwater snails) are present, and where the access to safe water and sanitation is limited (Figure 1). Infections can occur through recreational, habitual and employment activities of humans, and watering or grazing of animals. Human intestinal schistosomiasis symptoms range from abdominal pain, diarrhoea, blood in the stool, to liver and spleen enlargement, cancers and death (2). There is a dearth of data regarding $S$. japonicum and S. mekongi causing or not causing illness in animals.

The cornerstone of schistosomiasis control in all endemic areas is preventive chemotherapy with the anthelmintic praziquantel. However, drug treatment alone is likely insufficient to reach the elimination goal since only a few infected individuals are enough to maintain the transmission cycle (3). Significant gains towards $S$. japonicum elimination have been achieved with integrated intervention approaches (including preventive and selective chemotherapy, mollusciciding, health education, sanitation and environmental improvement) in countries such as China. Nevertheless, although transmission was interrupted in some provinces for over 10 years, other provinces remained endemic, and re-emergence was also observed in the last few years (4, 5, 6, 1). The progress towards control and elimination seems to be slowing down, with transmission still ongoing in many regions, and zoonotic schistosomiasis remaining a public health problem, particularly in the Philippines (7). In January 2021, schistosomiasis was targeted for elimination as a public health problem (EPHP) globally in the WHO's Road map for neglected tropical diseases 2021-2030 (8). This is achieved when the proportion of heavy intensity infections (over 400 eggs per gram of stool as per the Kato-Katz diagnostic) is reduced below $1 \%(8)$.

Zoonotic schistosome variants pose a unique challenge in achieving EPHP as they have multiple definitive host species contributing to transmission dynamics (9), which need to be more widely accounted for. Modelling efforts have largely concentrated on the more common non-zoonotic schistosomiasis, and have been successfully used to inform control strategies (10,11). The complexity of zoonotic schistosomiasis must be captured by these models if they are to continue to play a major role in public health policy (7). Here, we discuss the challenges of developing such models, and how a One-Health approach recognising the interconnection between people, animals, and their shared environment, can improve control programmes

\footnotetext{
${ }^{1}$ Who.int. 2021. Schistosomiasis. [online] Available at: https://www.who.int/news-room/fact-sheets/detail/schistosomiasis [Accessed 27 November 2021].

2 Who.int. 2021. Preventive chemotherapy (PC) data portal [online] Available at: https: //www . who. int/Global Healthobservatory [Accessed 27 November 2021].
} 
67 (12). Our vision builds on three cross-cutting pillars that we believe are vital to sustainable public health 68 policy, which we illustrate here in the context of zoonotic schistosomiasis EPHP; (1) understanding the 69 epidemiology, (2) economic considerations, and (3) accounting for sociological aspects.

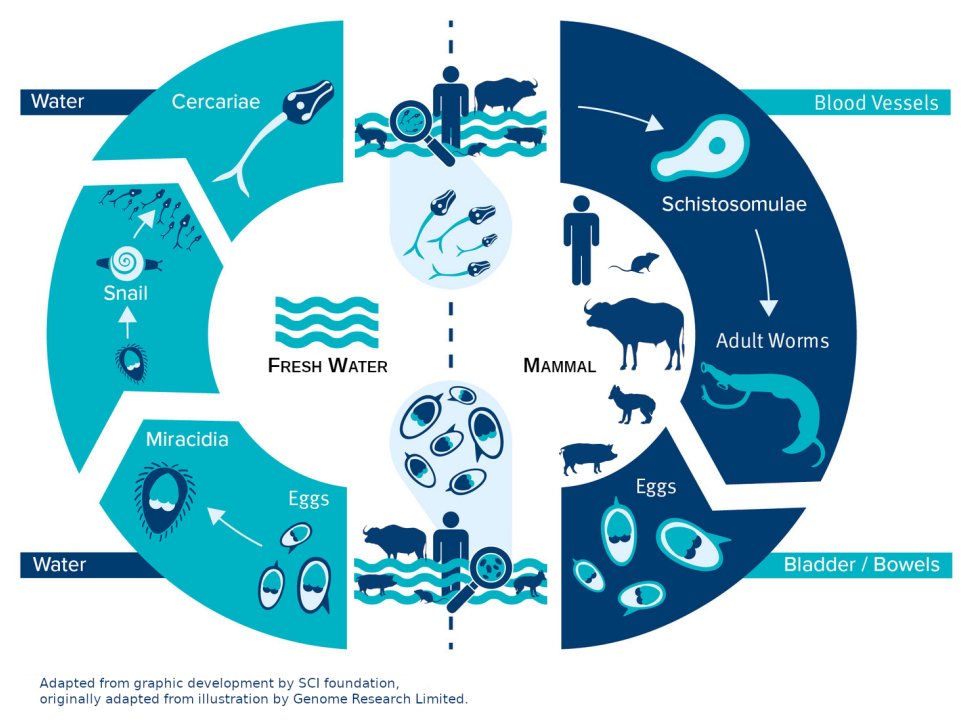

Figure 1. Life cycle of $\boldsymbol{S}$. japonicum and $\boldsymbol{S}$. mekongi. An infected definitive host, a mammal, passes schistosome eggs through the faeces into freshwater. The eggs may hatch into free-swimming larval miracidia that infect intermediate hosts, freshwater snails. After multiplication and development, snails shed free-swimming cercariae daily. The cercariae penetrate the skin of a mammal which comes in contact with water. Within the mammal, the cercariae shed their forked tail to form schistosomulae which mature and become worms. Paired male and female adult worms copulate and migrate to the mesenteric venules of the bowel and/or rectum where they lay thousands of eggs a day. Some of the eggs get back into the water through faeces and start the life cycle again, while some eggs get trapped in the organs causing disease symptoms.

\section{PILLAR I: EPIDEMIOLOGY}

This first pillar encompasses our understanding of the disease in its geographical context, including environmental drivers, host heterogeneity and parasite interactions. These can be informed through mathematical modelling which has provided quantitative evidence to inform intervention programmes and has played a vital role in informing the WHO's roadmap (13, 11). As a precursor to field trials, models ascertain long-term outcomes before implementing studies and control programmes, thus making them both more ethical and effective in the long-term. Analyses focused exclusively on this first pillar can identify the most effective strategies to achieve a target health outcome (14, 15, 16, 17).

In the context of communities where zoonotic schistosomiasis is endemic, livestock can be a major asset, residing closely with owners, fostering an environment where domestic animals may be responsible for a considerable amount of transmission to humans (18, 3). Infection dynamics in systems with multiple hosts differ significantly from single-host systems, and are therefore more complex to model. Transmission rates vary across definitive host species, which can be due to differing water contact behaviour, driving diversity in exposure and contamination rates. Similarly, each definitive host species will have different epidemiological characteristics in terms of recovery, birth, and mortality rates. Capturing definitive 
host heterogeneities in these disease dynamic processes translates to more accurately predicting higher prevalences and intensities of infection (19, 20).

Additionally, estimating the contribution of each definitive host species to parasite transmission is vital (13, 12). The best combination of interventions is expected to vary spatially according to animal host species' densities. For example, in China, attention had initially been exclusively on bovines, because historically, most of the research was conducted in lake areas where bovines were ubiquitous $(21,18,22,23)$. It is known today however, that various rodent species are the main animal hosts of S. japonicum in mountainous provinces, whilst bovines drive infection around the lakes $(24,3,25,26)$. The identification of locationspecific dominant animal reservoirs and their transmission contributions to humans, along with model calibration to local data are evidently crucial $(27,28)$. Quantifying each host's contribution to transmission enables the identification of maintenance and essential hosts, and the predicted impacts of control strategies targeting these hosts $(29,30,31)$. It has been shown that human-only treatment is insufficient to achieve EPHP because transmission is maintained by untreated reservoirs. Alternatively, interventions focused on the main reservoir predict success in reducing transmission to humans $(3,23,26)$.

Some modelling work has explored the importance of a range of animal and environmental controls (32, 33, 23, 31). Nonetheless, for models to provide reasonable insight, data collection needs to be improved. In particular, when human and animal data are collected independently, it becomes harder to consolidate and unify, hindering calibration and evaluation of multiple host models. As highlighted by WHO, providing centralised data access will facilitate and expedite analyses, and consequently the decision-making process (8). An example of this is the Pan-African Rabies Control Network (PARACON), which established a platform for centralised rabies data collection and analysis, improving on the WHO recommendation with an option for open data sharing (34, 35). We see a great opportunity in extending such a framework to other zoonotic diseases across a greater geographic area. These enhanced resources will enable improved models that capture the epidemiology of the disease and inform intervention effectiveness and timelines to reach programmatic goals.

\section{PILLAR II: ECONOMICS}

The second pillar accounts for the economic implications of, in this case, different interventions to achieve EPHP. For the implementation of an effective intervention strategy to be feasible, it must be affordable to individuals, governments and/or donors. This needs to include the generally high upfront start-up investment, as well as recurrent maintenance costs. A useful type of economic evaluation approach for this is cost-effectiveness analysis, where costs and non-monetary health effects of different control interventions can be compared (36). Results are expressed as additional costs per unit of improved health outcome, such as reduction in transmission rate, prevalence/incidence, or deaths (37). This type of analysis has already been used for numerous NTDs and can be integrated into highly detailed infection transmission models. In the context of zoonotic diseases such as $S$. japonicum and $S$. mekongi, it should be leveraged to explore the costs and effectiveness of animal-based and other combined interventions (38).

The costs that need to be considered are the expected resources used for implementing and eventually maintaining an intervention, including the net savings to patients and healthcare providers due to reducing the disease burden (36). Savings comprise out-of-pocket and health system expenses, travel costs of care seeking and opportunity costs of ill health, such as reduced patient productivity or school and work attendance, which are unfortunately often overlooked. In zoonotic schistosomiasis, additional costs are 
124

incurred due to animal death or illness, which adds time and cost to replace these animals, as well as reduced livestock productivity in quantity and/or quality (39).

One challenge remains regarding the appropriate metric to use for health outcomes that is generalizable. Disability-adjusted life-years (DALYs) are widely used to measure disease burden, with one DALY representing the loss of one life-year lived in optimal health, thereby translating both the disease mortality (the years of life lost, YLL), and morbidity (the years lived with disability, YLD) into a single metric (40, 41). This enables comparison across studies, settings, and interventions targeting the same or different diseases (37). However, DALYs face some limitations, as they frequently disregard the infection-associated mental health burden or the need to adjust for co-morbidities. To estimate morbidity, DALYs rely on general estimates of disability weights - most of them estimated by an expert medical panel, instead of a preferencebased valuation method, raising universality concerns (42). This leads to the health impacts of infection being underestimated. Lastly, DALYs are unsuitable when evaluating zoonotic diseases because they disregard the effectiveness resulting from improving animals' and owners' quality of life and well-being due to averted animal morbidity/mortality.

Efforts have been made to quantify the zoonosis burden on humans and animals simultaneously - the zoonosis disability-adjusted life-years (zDALYs) (43). These include an additional component called animal loss equivalents, which converts the expected livestock production and local per capita income losses to the equivalent number of human YLD. Nevertheless, this metric has been rarely used in cost-effectiveness analyses of zoonotic diseases (44, 45), and only once for schistosomiasis (46).

Most economic evaluations of schistosomiasis interventions have focused on chemotherapy with praziquantel (47, 48, 49). The WHO NTD roadmap has highlighted the benefits, including financial, of cross-cutting interventions (8). However, the use of different effectiveness measures (i.e. health outcomes) for the evaluation of new control interventions hampers the comparison within and between NTDs (50, 51). Therefore, standardising the use of a common metric across economic analyses will enable cost-effectiveness comparisons across multiple NTDs. Such metrics should be extendable, as appropriate to zoonotic diseases. Programmes that consider the first and second pillars together are more sustainable, as knowledge of the most effective interventions from the epidemiological perspective can be supplemented by evaluations of their likely economic impact.

\section{PILLAR III: SOCIOLOGY}

The third pillar takes into consideration the impact of human behaviour on intervention outcome. This is most commonly considered in the context of adherence, which refers to the way in which individuals interact with a given intervention, for example, when offered medication, whether a person will ingest it or not - an important differentiation between treatment coverage and treatment compliance $(52,53)$. In the context of zoonotic schistosomiasis, this can also refer to whether a person does indeed treat their animals. If enough individuals do not adhere to control measures, the effectiveness at the population level can be lower than predicted. This can lead to failure in meeting targets like EPHP and/or increases in intervention costs, which in turn, could change the expected cost-effectiveness of the programme. Unfortunately, but not surprisingly, numerous studies across diseases (not just NTDs) suggest that it is not realistic to expect full compliance with any control measure $(54,55,56,57,58)$. Intervention strategies can be better informed when models explicitly account for inconsistent adherers (59, 53).

Assessing the impact of novel interventions in a given location can be challenging when the social determinants of participation are unknown. In the context of NTDs, the WHO has suggested that Water, 
165 Sanitation, and Hygiene (WaSH) methods should be incorporated into all programmes (8). This will be

\section{DISCUSSION}

181 Sustainability has been discussed in many fields, and always encompasses three pillars, environmental, 182

\section{CONFLICT OF INTEREST STATEMENT}

198 The authors declare that the research was conducted in the absence of any commercial or financial 199

\section{AUTHOR CONTRIBUTIONS}

200 E. J. and J. M. P. conceived the study. E. J., J. C., O. K. wrote the original draft. All authors critically 201 economic, and social. Here, we have linked those three pillars in the context of NTDs, for the purpose of informing sustainable global health policy. This is of particular importance in the case of zoonotic NTDs (like schistosomiasis caused by S. japonicum and S. mekongi), where there is a need to recognise the interconnection between people, animals, and their shared environment - emphasising the importance of a One-Health approach. For the first pillar, epidemiology, we focus on definitive host heterogeneity and quantifying their contribution to overall transmission, enabling us to inform optimal intervention strategies and timelines to programmatic targets. Epidemiological understanding will be strengthened through access to improved cross-disease data. The second pillar, economics, can inform interventions by accounting for costs to all stakeholders involved and the time horizon for cost benefits. Challenges remain in establishing a standardised metric for cost-effectiveness evaluation that is appropriate for zoonotic diseases. The third, and last, pillar, Social, considers the impact of human behaviour - particularly adherence - on programmatic success. Only by combining these three pillars can we develop the best strategies that achieve the desired target health outcomes, whilst considering costs, that will be championed by relevant stakeholders approved by local communities. The modelling community may use these recommendations to make the model predictions more accurate, assisting decision-makers to design sustainable control programmes to reach the WHO's ambitious 2030 goal. relationships that could be construed as a potential conflict of interest. 


\section{FUNDING}

This work was supported by the European Research Council (starting grant SCHISTO_PERSIST_680088 awarded to P. H. L. L., supporting J. C.); Wellcome Trust (grant 204820/Z/16/Z awarded to P. H. L. L., supporting J. C.); the Engineering and Physical Sciences Research Council (grants EP/T003618/1 to E. J., S. A., P. H. L. L., J. M. P.); the Drugs for Neglected Diseases Initiative (grant awarded to P. H. L. L., supporting J. C.); the Medical Research Council (grant MR/P025447/1 to P. H. L. L., supporting J. C.) and the Newton Fund awarded through the Medical Research Council (MRC; grant number MR/R025592/1 to O. K., M. B. and J. M. P.).

\section{REFERENCES}

1.Gordon CA, Kurscheid J, Williams GM, Clements ACA, Li Y, Zhou XN, et al. Asian Schistosomiasis: Current Status and Prospects for Control Leading to Elimination. Tropical Medicine and Infectious Disease 4 (2019) 40. doi:10.3390/tropicalmed4010040. Number: 1 Publisher: Multidisciplinary Digital Publishing Institute.

2 .Gray DJ, Ross AG, Li YS, McManus DP. Diagnosis and management of schistosomiasis. BMJ 342 (2011) d2651. doi:10.1136/bmj.d2651. Publisher: British Medical Journal Publishing Group Section: Clinical Review.

3 .Rudge JW, Webster JP, Lu DB, Wang TP, Fang GR, Basáñez MG. Identifying host species driving transmission of schistosomiasis japonica, a multihost parasite system, in China. PNAS 110 (2013) 11457-11462. doi:10.1073/pnas.1221509110. Publisher: National Academy of Sciences Section: Biological Sciences.

4.Xiang J, Chen H, Ishikawa H. A mathematical model for the transmission of Schistosoma japonicum in consideration of seasonal water level fluctuations of Poyang Lake in Jiangxi, China. Parasitology international 62 (2013) 118-126.

5.Zhu HR, Liu L, Zhou XN, Yang GJ. Ecological model to predict potential habitats of oncomelania hupensis, the intermediate host of schistosoma japonicum in the mountainous regions, china. PLoS neglected tropical diseases 9 (2015) e0004028.

6.World Health Organization and others. Expert consultation to accelerate elimination of asian schistosomiasis, shanghai, china, 22-23 may 2017: meeting report. Tech. rep., Manila: WHO Regional Office for the Western Pacific (2017).

7 .Betson M, Alonte AJI, Ancog RC, Aquino AMO, Belizario VY, Bordado AMD, et al. Chapter Two Zoonotic transmission of intestinal helminths in southeast Asia: Implications for control and elimination. Rollinson D, Stothard R, editors, Advances in Parasitology (Academic Press), vol. 108 (2020), 47-131. doi:10.1016/bs.apar.2020.01.036.

8.WHO. Ending the neglect to attain the Sustainable Development Goals: a road map for neglected tropical diseases 2021-2030. (2020).

9.Webster JP, Borlase A, Rudge JW. Who acquires infection from whom and how? Disentangling multi-host and multi-mode transmission dynamics in the 'elimination' era. Philosophical Transactions of the Royal Society B: Biological Sciences 372 (2017) 20160091. doi:10.1098/rstb.2016.0091.

10 .Consortium NM, of Control of Neglected Tropical Diseases WD. Achieving NTD Control, Elimination and Eradication Targets Post-2020 Modelling Perspectives and Priorities. Gates Open Res 3 (2019) 1664. doi:10.21955/gatesopenres.1116563.1. Number: 1664 Publisher: F1000 Research Limited. 
11 .Clark J, Stolk WA, Basáñez MG, Coffeng LE, Cucunubá ZM, Dixon MA, et al. How modelling can help steer the course set by the World Health Organization 2021-2030 roadmap on neglected tropical diseases. Gates Open Res 5 (2021) 112. doi:10.12688/gatesopenres.13327.1.

12 .Laing G, Vigilato MAN, Cleaveland S, Thumbi SM, Blumberg L, Salahuddin N, et al. One Health for neglected tropical diseases. Transactions of The Royal Society of Tropical Medicine and Hygiene 115 (2021) 182-184. doi:10.1093/trstmh/traa117.

13 .NTD Modelling Consortium Schistosomiasis Group. Insights from quantitative and mathematical modelling on the proposed WHO 2030 goal for schistosomiasis. Gates Open Res 3 (2019) 1517. doi:10.12688/gatesopenres.13052.2.

14.Farrell SH, Coffeng LE, Truscott JE, Werkman M, Toor J, de Vlas SJ, et al. Investigating the Effectiveness of Current and Modified World Health Organization Guidelines for the Control of SoilTransmitted Helminth Infections. Clinical Infectious Diseases 66 (2018) S253-S259. doi:10.1093/cid/ ciy002.

15 .Toor J, Alsallaq R, Truscott JE, Turner HC, Werkman M, Gurarie D, et al. Are We on Our Way to Achieving the 2020 Goals for Schistosomiasis Morbidity Control Using Current World Health Organization Guidelines? Clinical Infectious Diseases 66 (2018) S245-S252. doi:10.1093/cid/ciy001.

16.Kura K, Ayabina D, Toor J, Hollingsworth TD, Anderson RM. Disruptions to schistosomiasis programmes due to COVID-19: an analysis of potential impact and mitigation strategies. Transactions of The Royal Society of Tropical Medicine and Hygiene 115 (2021) 236-244. doi:10.1093/trstmh/traa202.

17 .Toor J, Adams ER, Aliee M, Amoah B, Anderson RM, Ayabina D, et al. Predicted Impact of COVID19 on Neglected Tropical Disease Programs and the Opportunity for Innovation. Clinical Infectious Diseases 72 (2021) 1463-1466. doi:10.1093/cid/ciaa933.

18 .Guo J, Li Y, Gray D, Ning A, Hu G, Chen H, et al. A DRUG-BASED INTERVENTION STUDY ON THE IMPORTANCE OF BUFFALOES FOR HUMAN SCHISTOSOMA JAPONICUM INFECTION AROUND POYANG LAKE, PEOPLE'S REPUBLIC OF CHINA. The American Journal of Tropical Medicine and Hygiene 74 (2006) 335-341. doi:10.4269/ajtmh.2006.74.335. Publisher: The American Society of Tropical Medicine and Hygiene.

19 .Barbour AD. Modelling the transmission of schistosomiasis: an introductory view. 55 (1996) 135-143. 20 .Mari L, Ciddio M, Casagrandi R, Perez-Saez J, Bertuzzo E, Rinaldo A, et al. Heterogeneity in schistosomiasis transmission dynamics. Journal of Theoretical Biology 432 (2017) 87-99. doi:10. 1016/j.jtbi.2017.08.015.

21.Williams GM, Sleigh AC, Li Y, Feng Z, Davis GM, Chen H, et al. Mathematical modelling of schistosomiasis japonica: comparison of control strategies in the People's Republic of China. Acta Tropica 82 (2002) 253-262. doi:10.1016/S0001-706X(02)00017-7.

22 .Gao SJ, Cao HH, He YY, Liu YJ, Zhang XY, Yang GJ, et al. The basic reproductive ratio of Barbour's two-host schistosomiasis model with seasonal fluctuations. Parasites Vectors 10 (2017) 42. doi:10.1186/s13071-017-1983-1.

23.Williams GM, Li Ys, Gray DJ, et al, McManus DP. Field Testing Integrated Interventions for Schistosomiasis Elimination in the People's Republic of China: Outcomes of a Multifactorial ClusterRandomized Controlled Trial (2019).

24 .Seto EYW, Remais JV, et al, Zhou XN. Toward Sustainable and Comprehensive Control of Schistosomiasis in China: Lessons from Sichuan (2011).

25.Webster JP, Gower CM, Knowles SCL, Molyneux DH, Fenton A. One health - an ecological and evolutionary framework for tackling Neglected Zoonotic Diseases. Evolutionary Applications 9 (2016) 313-333. doi:10.1111/eva.12341. _eprint: https://onlinelibrary.wiley.com/doi/pdf/10.1111/eva.12341. 
26 .Zou HY, Yu QF, Qiu C, Webster JP, Lu DB. Meta-analyses of Schistosoma japonicum infections in wild rodents across China over time indicates a potential challenge to the 2030 elimination targets. PLOS Neglected Tropical Diseases 14 (2020) e0008652. doi:10.1371/journal.pntd.0008652. Publisher: Public Library of Science.

27 .Streicker DG, Fenton A, Pedersen AB. Differential sources of host species heterogeneity influence the transmission and control of multihost parasites. Ecology Letters 16 (2013) 975-984. doi:10.1111/ele. 12122. _eprint: https://onlinelibrary.wiley.com/doi/pdf/10.1111/ele.12122.

28 .Fenton A, Streicker DG, Petchey OL, Pedersen AB. Are All Hosts Created Equal? Partitioning Host Species Contributions to Parasite Persistence in Multihost Communities. The American Naturalist 186 (2015) 610-622. doi:10.1086/683173. Publisher: The University of Chicago Press.

29 .Bielby J, Price SJ, Monsalve-CarcaÑo C, Bosch J. Host contribution to parasite persistence is consistent between parasites and over time, but varies spatially. Ecological Applications 31 (2021) e02256. doi:10.1002/eap.2256. _eprint: https://onlinelibrary.wiley.com/doi/pdf/10.1002/eap.2256.

30 .Erazo D, Pedersen AB, Gallagher K, Fenton A. Who acquires infection from whom? Estimating herpesvirus transmission rates between wild rodent host groups. Epidemics 35 (2021) 100451. doi:10. 1016/j.epidem.2021.100451.

31 .Borlase A, Rudge JW, Léger E, Diouf ND, Fall CB, Diop SD, et al. Spillover, hybridization, and persistence in schistosome transmission dynamics at the human-animal interface. PNAS 118 (2021). doi:10.1073/pnas.2110711118. Publisher: National Academy of Sciences Section: Biological Sciences.

32 .Hisakane N, Kirinoki M, Chigusa Y, Sinuon M, Socheat D, Matsuda H, et al. The evaluation of control measures against Schistosoma mekongi in Cambodia by a mathematical model. Parasitology International 57 (2008) 379-385. doi:10.1016/j.parint.2008.03.003.

33 .Dong Y, Du CH, Zhang Y, Wang LF, Song J, Wu MS, et al. Role of ecological approaches to eliminating schistosomiasis in Eryuan County evaluated by system modelling. Infectious Diseases of Poverty 7 (2018) 129. doi:10.1186/s40249-018-0511-7.

34 .Scott TP, Coetzer A, de Balogh K, Wright N, Nel LH. The Pan-African Rabies Control Network (PARACON): A unified approach to eliminating canine rabies in Africa. Antiviral Research 124 (2015) 93-100. doi:10.1016/j.antiviral.2015.10.002.

35 .Haselbeck AH, Rietmann S, Tadesse BT, Kling K, Kaschubat-Dieudonné ME, Marks F, et al. Challenges to the Fight against Rabies-The Landscape of Policy and Prevention Strategies in Africa. International Journal of Environmental Research and Public Health 18 (2021) 1736. doi:10.3390/ijerph18041736. Number: 4 Publisher: Multidisciplinary Digital Publishing Institute.

36 .Drummond MF, Sculpher MJ, Claxton K, Stoddart GL, Torrance GW. Methods for the Economic Evaluation of Health Care Programmes (Oxford University Press) (2015). Google-Books-ID: yzZSCwAAQBAJ.

37 .Turner HC, French MD, Montresor A, King CH, Rollinson D, Toor J. Economic evaluations of human schistosomiasis interventions: a systematic review and identification of associated research needs. Wellcome Open Res 5 (2020) 45. doi:10.12688/wellcomeopenres.15754.2.

38 .Adeyemo P, Léger E, Hollenberg E, Diouf N, Sene M, Webster JP, et al. Estimating the Financial Impact of Livestock Schistosomiasis on Traditional Subsistence and Transhumance Farmers Keeping Cattle, Sheep and Goats in Northern Senegal. preprint, In Review (2021). doi:10.21203/rs.3.rs-757540/v1.

39 .Narrod C, Zinsstag J, Tiongco M. A one health framework for estimating the economic costs of zoonotic diseases on society. Ecohealth 9 (2012) 150-162. doi:10.1007/s10393-012-0747-9.

40 .Murray CJ, Lopez AD, Jamison DT. The global burden of disease in 1990: summary results, sensitivity analysis and future directions. Bull World Health Organ 72 (1994) 495-509. 
41 .Ung L, Stothard JR, Phalkey R, Azman AS, Chodosh J, Hanage WP, et al. Towards global control of parasitic diseases in the Covid-19 era: One Health and the future of multisectoral global health governance. Adv Parasitol 114 (2021) 1-26. doi:10.1016/bs.apar.2021.08.007.

42 .Chen A, Jacobsen KH, Deshmukh AA, Cantor SB. The evolution of the disability-adjusted life year (DALY). Socio-Economic Planning Sciences 49 (2015) 10-15. doi:10.1016/j.seps.2014.12.002.

43 .Torgerson PR, Rüegg S, Devleesschauwer B, Abela-Ridder B, Havelaar AH, Shaw APM, et al. zDALY: An adjusted indicator to estimate the burden of zoonotic diseases. One Health 5 (2018) 40-45. doi:10.1016/j.onehlt.2017.11.003.

44 .Okello WO, Okello AL, Inthavong P, Tiemann T, Phengsivalouk A, Devleesschauwer B, et al. Improved methods to capture the total societal benefits of zoonotic disease control: Demonstrating the costeffectiveness of an integrated control programme for Taenia solium, soil transmitted helminths and classical swine fever in northern Lao PDR. PLOS Neglected Tropical Diseases 12 (2018) e0006782. doi:10.1371/journal.pntd.0006782. Publisher: Public Library of Science.

45 .Saadi A, Amarir F, Filali H, Thys S, Rhalem A, Kirschvink N, et al. The socio-economic burden of cystic echinococcosis in Morocco: A combination of estimation method. PLOS Neglected Tropical Diseases 14 (2020) e0008410. doi:10.1371/journal.pntd.0008410. Publisher: Public Library of Science.

46 .Zayas LPN, Rüegg S, Torgerson P. The burden of zoonoses in Paraguay: A systematic review. PLOS Neglected Tropical Diseases 15 (2021) e0009909. doi:10.1371/journal.pntd.0009909. Publisher: Public Library of Science.

47 .Tan D, Yu D, Li Y, Xie MS, Wen HM, Cai WB, et al. Analysis of Cost-Effectiveness on Two Chemotherapy Schemes to Schistosomiasis in Hyperendemic Villages of Hunan Province. Practical Preventire Medicine (2001).

48 .Yu D, Jr JNS, Hutton G, Tan D, Tanner M. COST-EFFECTIVENESS ANALYSIS OF THE IMPACTS ON INFECTION AND MORBIDITY ATTRIBUTABLE TO THREE CHEMOTHERAPY SCHEMES AGAINST SCHISTOSOMA JAPONICUM IN HYPERENDEMIC AREAS OF THE DONGTING LAKE REGION, CHINA. SOUTHEAST ASIAN J TROP MED PUBLIC HEALTH 33 (2002) 17.

49 .Croce D, Porazzi E, Foglia E, Restelli U, Sinuon M, Socheat D, et al. Cost-effectiveness of a successful schistosomiasis control programme in Cambodia (1995-2006). Acta Tropica 113 (2010) 279-284. doi:10.1016/j.actatropica.2009.11.011.

50 .Zhang Hm, Yu Q, Zhang X, Coa Cl, Li Sz, Zhu H. [Cost-effectiveness evaluation on comprehensive control measures carrying out in schistosomiasis endemic areas with regard to different layers of administrative villages stratified by infection situation of human and domestic animals. I. Costeffectiveness study in inner embankment of marshland and lake regions from 2006 to 2010]. Zhongguo Xue Xi Chong Bing Fang Zhi Za Zhi 26 (2014) 254-259.

51 .Ya Y, Jian-Bing L, Hao L, Shi-Gui L, Zhong H, Bin C, et al. [Cost-effectiveness of comprehensive schistosomiasis control strategy with focus on cattle and sheep removal in Junshan District, Yueyang City]. Zhongguo Xue Xi Chong Bing Fang Zhi Za Zhi 30 (2017) 14-17. doi:10.16250/j.32.1374. 2017136.

52 .Adriko M, Faust CL, Carruthers LV, Moses A, Tukahebwa EM, Lamberton PHL. Low Praziquantel Treatment Coverage for Schistosoma mansoni in Mayuge District, Uganda, Due to the Absence of Treatment Opportunities, Rather Than Systematic Non-Compliance. Tropical Medicine and Infectious Disease 3 (2018) 111. doi:10.3390/tropicalmed3040111. Number: 4 Publisher: Multidisciplinary Digital Publishing Institute. 
53 .Graham M, Ayabina D, Lucas TC, Collyer BS, Medley GF, Hollingsworth TD, et al. SCHISTOX: An individual based model for the epidemiology and control of schistosomiasis. Infectious Disease Modelling 6 (2021) 438-447. doi:10.1016/j.idm.2021.01.010.

54 .Babu BV, Babu GR. Coverage of, and compliance with, mass drug administration under the programme to eliminate lymphatic filariasis in India: a systematic review. Transactions of The Royal Society of Tropical Medicine and Hygiene 108 (2014) 538-549. doi:10.1093/trstmh/tru057.

55 .Chami GF, Kontoleon AA, Bulte E, Fenwick A, Kabatereine NB, Tukahebwa EM, et al. Communitydirected mass drug administration is undermined by status seeking in friendship networks and inadequate trust in health advice networks. Social Science \& Medicine 183 (2017) 37-47. doi:10.1016/j.socscimed.2017.04.009.

56 .Inobaya MT, Chau TN, Ng SK, MacDougall C, Olveda RM, Tallo VL, et al. Mass drug administration and the sustainable control of schistosomiasis: an evaluation of treatment compliance in the rural Philippines. Parasites Vectors 11 (2018) 1-11. doi:10.1186/s13071-018-3022-2. Number: 1 Publisher: BioMed Central.

57 .Chami GF, Bundy DAP. More medicines alone cannot ensure the treatment of neglected tropical diseases. The Lancet Infectious Diseases 19 (2019) e330-e336. doi:10.1016/S1473-3099(19)30160-4.

58.Cahapay MB. To get or not to get: Examining the intentions of Philippine teachers to vaccinate against COVID-19. Journal of Human Behavior in the Social Environment 0 (2021) 1-11. doi:10.1080/10911359.2021.1896409. Publisher: Routledge _eprint: https://doi.org/10.1080/10911359.2021.1896409.

59 .Dyson L, Stolk WA, Farrell SH, Hollingsworth TD. Measuring and modelling the effects of systematic non-adherence to mass drug administration. Epidemics 18 (2017) 56-66. doi:10.1016/j.epidem.2017. 02.002 .

60. Wood S, Sawyer R, Simpson-Hébert M. Participatory Hygiene and Sanitation Transformation Initiative (PHAST) step by step guide: a participatory approach for the control of diarrhoeal disease (World Health Organization) (1998).

61 .Kosinski KC, Kulinkina AV, Abrah AFA, Adjei MN, Breen KM, Chaudhry HM, et al. A mixed-methods approach to understanding water use and water infrastructure in a schistosomiasis-endemic community: case study of Asamama, Ghana. BMC Public Health 16 (2016) 322. doi:10.1186/s12889-016-2976-2.

62 .Meginnis K, Hanley N, Mujumbusi L, Lamberton PHL. Non-monetary numeraires: Varying the payment vehicle in a choice experiment for health interventions in Uganda. Ecological Economics 170 (2020) 106569. doi:10.1016/j.ecolecon.2019.106569. 